\title{
A new concept for experiment program planning for the fusion experiment Wendelstein 7-X
}

\author{
J. Schacht, H. Laqua, M. Lewerentz, A. Spring
}

\begin{abstract}
The control and data acquisition system (codac system) for the fusion experiment Wendelstein $7-X$ is designed for miscellaneous operational scenarios: short pulses with arbitrary intervals, steady-state long pulse operation and arbitrary sequences of short phases with different characteristics in one discharge. Due to the multitude and diversity of the necessary settings of an experiment program special attention must be paid to experiment planning. The reduction of the editable parameters depending dedicated to the particular experiment task is an important requirement. Furthermore experiment planners prefer a more abstract and physics-oriented description.

This contribution introduces a concept for experiment planning based on so called segment program boxes (SegProgBox). The planned work flow of experiment program creation will be discussed after an introduction of the SegProgBox design.
\end{abstract}

Index Terms - control system, distributed real time system, fusion experiment, experiment program editing.

\section{INTRODUCTION}

$\mathrm{T}$ HE super conducting fusion experiment Wendelstein 7-X (W7-X) is designed for long pulse and steady state operation with plasma discharge duration up to $30 \mathrm{~min}$ with full heating power. The assembly phase of the machine W7-X and its components, like heating systems, cooling systems, cryogenic systems and diagnostics is still running and will be finished in 2014.

With respect to the advanced capabilities of $\mathrm{W} 7-\mathrm{X}$ new control and data acquisition concepts [1][2][3] have been designed and tested successfully [4][5][6]. The complex W7$\mathrm{X}$ control system with a large number of technical and scientific components will operate in a widely automatic way.

Due to the multitude and diversity of the necessary settings which describe an experiment run special attention must be paid to experiment planning. The reduction of editable parameters adequate to the respective experiment task is an important requirement. Furthermore experiment planners prefer a more abstract and physics-oriented description for planning and editing of experiments.

Manuscript received Mai, 2009.

J. Schacht, H. Laqua, M. Lewerentz, and A. Spring: Max-Planck Institute für Plasmaphysik, Teilinstitut Greifswald, D-17489 Greifswald, Germany. (email: joerg.schacht@ipp.mpg.de).

\section{SEGMENT CONTROL SYSTEM}

During experiment operation all technical components (e.g. heating systems, cooling systems, magnet system) and necessary diagnostics are subordinated to the central control system. The segment control system of a component (also called group) consists of one or more computers called control stations. The control stations contain modules for controlling sensors and actors and other tasks (e.g. modules for segment control, data processing, message processing). During a W7-X experiment run local segment controllers of the components are co-ordinated by the central sequence controller (cSegSC), which is part of the central control system. When controlled centrally the different components (groups) work together in a so called project.

An experiment run is defined by an experiment program. The smallest entity of an experiment program is a so called segment. A segment description reflects the hierarchical structure of the W7-X control system and is a complete set of parameters of all participating modules.

A configuration is an aggregation of the software modules implemented in a control station. On the next higher level references to the station configuration can be found. On the highest level the project configuration is located. It describes the participating components (fig. 1). The configuration information is stored in an object oriented data base.

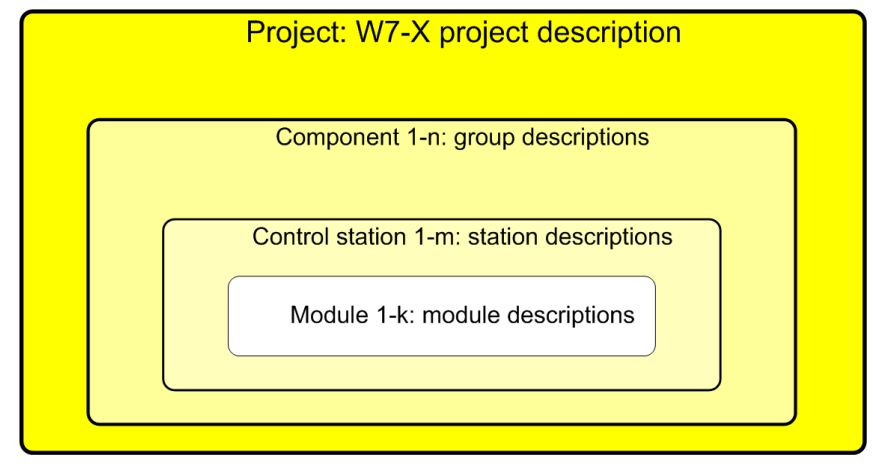

Fig. 1: Hierarchy of W7-X configuration

The configuration contains all necessary information to create a default segment. For special use cases so called pattern segment descriptions can be defined. A pattern segment description contains a complete set of user defined 
low level parameters for all modules of a component. Low level parameters (LLP) are the representation of real technical parameters of the modules used to control the behavior during segment execution.

One has to differentiate between project segments, if the experiment is centrally controlled and group segment, if a component is in autonomous mode. Project segments completely describe the behavior of all participating components of the fusion device during the concerning period. They are an aggregation of the respective group segments. An experiment program consists of a chain of segments. The central segment sequence controller is responsible to process the chain of segments, which is arranged by the experiment leader. After the program execution is started, the operator (manually) or dedicated states of the modules or of the plasma (automatically) can change the predefined sequence of segments of the experiment program (see fig. 2).

Segment programs can be used for the operation of the whole W7-X device or for commissioning and testing of a single component in autonomous control mode.

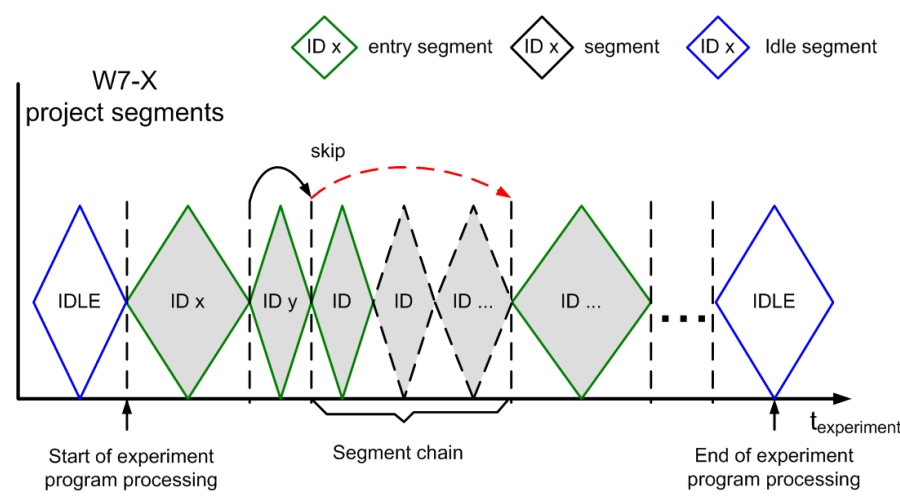

Fig. 2. Segment processing

Often the number of low level segment parameters is quite large. To reduce complexity and to open the opportunity of a more abstract physics-oriented description a higher description level was introduced. Therefore a set of high level parameters (HLP) can be defined for a component, which is transformed to a subset of the technical parameters by using transformation functions [3]. Such a set is called high level (group) segment.

\section{EXPERIMENT PROGRAM PLANNING}

Due to the multitude and diversity of the necessary settings special attention must be paid to experiment planning. The main task of experiment planning is to define both the content (structure and parameterization) of segments and the sequence of segments. Necessary input information for this planning process are a detailed description (proposals) of the experiment given by the requester, the actual machine configuration and a set of rules and conditions for the usage of components and its elements.

The result of the planning process is a sequence of complete segment descriptions for the whole project including executable low level segments. A sequence of segments can cover an entire experiment run or only parts of it.

Reduction the amount of editable parameters adequate to the respective experiment task is an important requirement. Often there are recurring behavior patterns in experiment programs. The edit process could be faster, easier to handle, well-arranged, and more reliable if a description of these behaviors could be done task specific and component comprehensive. A next important requirement is the possibility to use and integrate gained experiences from experiments regarding rules, dependencies and sequences in the experiment planning process.

In the next chapter a new concept for experiment planning based on so called Segment Program Boxes (SegProgBox) will be introduced.

\section{THE SEgProgBox CONCEPT}

SegProgBoxes are special user defined data structures, which are presented to the user as smallest entity to built experiment programs from. A SegProgBox defines the behavior on a task oriented view of one or more of the segment controlled components of a project like W7-X. It is also possible that a SegProgBox contains the complete set of the project components. The vertical dimension (y-axis) of a SegProgBox is set by the number of included components.

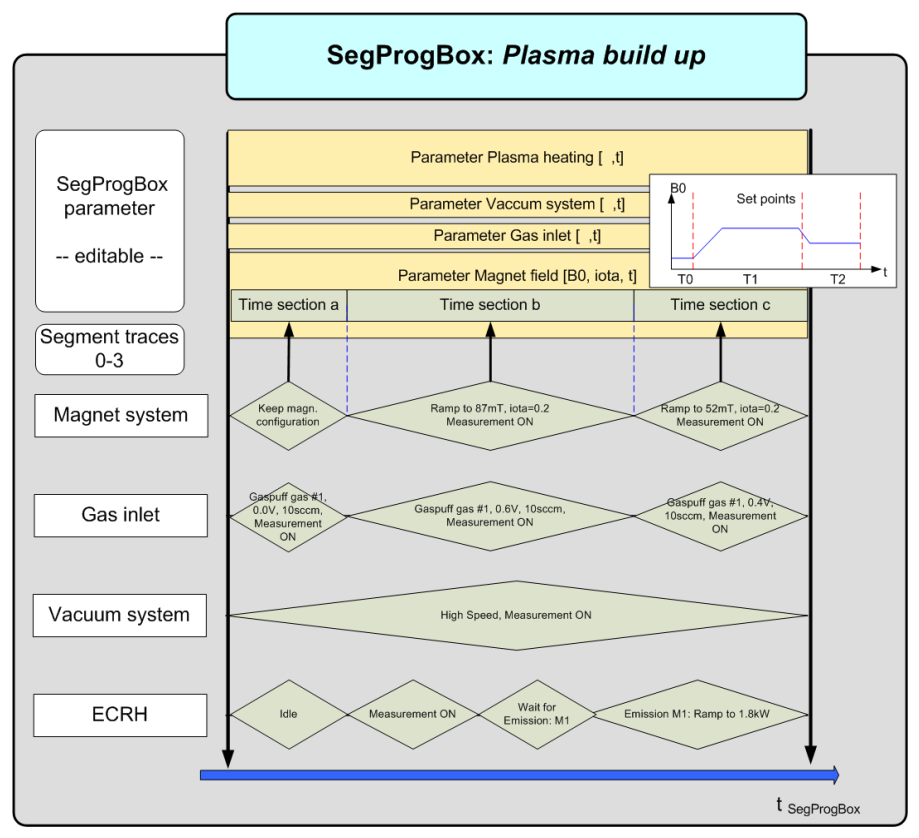

Fig. 3: Example for a SegProgBox configuration for plasma build up

For every component included in a SegProgBox an own time trace is used, which is called segment trace. A segment trace has a given time period and a time grid. For W7-X project this time grid has the same value as the shortest segment duration and is therefore set to $10 \mathrm{~ms}$. The horizontal dimension (x-axis) of a SegProgBox is equivalent to the time axis and represents the time period of a SegProgBox. 
Distension or compression of the horizontal SegProgBox dimension changes the activity interval and time-scales the SegProgBox. The minimal value for the duration of a SegProgBox is equal to the minimal time interval of a segment (10 ms for W7-X project). The maximal activity length of a SegProgBox is another time property. The behavior of the segments of the segment traces by time-scaling operations can be set by rules. These rules define which part of a segment trace are fixed or can be scaled by using a given time-scaling function.

For each assigned component the corresponding segment trace contains at least one or more High Level group segments.

These segments are arranged in such a manner, that the requested changes of the components' behavior will be realized. In the context of High Level segments of the other segment traces task oriented sequences for a set of components are defined.

Fig. 3 shows an example for a SegProgBox configuration. The application for this SegProgBox is the definition of the behavior of the components magnet supplies, gas inlet, vacuum system and plasma heating system during the plasma build up phase at stellarator WEGA. The given segment sequences assures the necessary sequence of components operations with an adequate set of parameters for ignition of a plasma (this example is related to the stellarator WEGA [6]).

Only fully defined High Level segments with appropriate Low Level segments and transformation functions can be integrated into a SegProgBox.

A SegProgBox can be parameterized by a set of standard and individual parameters. These parameters can be divided into editable or non-editable (but visible) parameters.

The different types of SegProgBox parameters are summarized in tab. 1.

\begin{tabular}{|c|c|c|}
\hline $\begin{array}{c}\text { parameter } \\
\text { type }\end{array}$ & usage & example \\
\hline $\begin{array}{l}\text { general } \\
\text { parameters }\end{array}$ & $\begin{array}{l}\text { - identification of } \\
\text { SegProgBox, } \\
\text { - project assignment, } \\
\text { - configuration } \\
\text { information for included } \\
\text { components, } \\
\text { - description of content } \\
\text { and behavior of } \\
\text { SegProgBox, }\end{array}$ & $\begin{array}{l}\text { SegProgBox_ID, } \\
\text { Project_ID, }\end{array}$ \\
\hline $\begin{array}{l}\text { time } \\
\text { parameters }\end{array}$ & $\begin{array}{l}\text { - default values for } \\
\text { duration of } \\
\text { SegProgBox, } \\
\text { - time scaling behavior, } \\
\text { - minimum and } \\
\text { maximum values for } \\
\text { duration, }\end{array}$ & $\begin{array}{l}\mathrm{t}_{\min }=200 \mathrm{~ms}, \\
\mathrm{t}_{\max }=100 \mathrm{~s}, \\
\mathrm{t}_{\text {default }}=500 \mathrm{~ms},\end{array}$ \\
\hline $\begin{array}{l}\text { High Level } \\
\text { Parameters of } \\
\text { SegProgBox }\end{array}$ & $\begin{array}{l}\text { - Definition of the } \\
\text { control behavior of the } \\
\text { components of the } \\
\text { SegProgBox (can be set } \\
\text { as editable or as fixed), } \\
\text { - values of characteristic } \\
\text { parameters can be }\end{array}$ & $\begin{array}{l}\text { magnetic } \\
\text { configuration } \mathrm{xx}\left[\mathrm{p}_{0^{-}}\right. \\
\left.\mathrm{p}_{\mathrm{m}}\right] \\
\text { ECRH heating } \\
\text { scenario yy }\left[\mathrm{p}_{0}-\mathrm{p}_{\mathrm{n}}\right] \text {, }\end{array}$ \\
\hline
\end{tabular}

\begin{tabular}{|c|c|c|}
\hline & $\begin{array}{l}\text { changed during } \\
\text { experiment program } \\
\text { planning, }\end{array}$ & \\
\hline $\begin{array}{l}\text { High Level } \\
\text { Parameters of } \\
\text { included } \\
\text { components }\end{array}$ & $\begin{array}{l}\text { - internal parameters, } \\
\text { given by the HLP } \\
\text { segments of the } \\
\text { components segment } \\
\text { traces, } \\
\text { - will be set by the High } \\
\text { Level Parameters of the } \\
\text { SegProgBox by using } \\
\text { transformation } \\
\text { functions }\end{array}$ & $\begin{array}{l}\text { magnet field strength } \\
\mathrm{B}_{\text {axis, }} \\
\text { iota, shear, }\end{array}$ \\
\hline $\begin{array}{l}\text { properties for } \\
\text { edit and } \\
\text { simulation } \\
\text { purposes }\end{array}$ & $\begin{array}{l}\text { - HLPs of a } \\
\text { SegProgBox are } \\
\text { connected to a set of } \\
\text { parameters to define the } \\
\text { permitted ranges and for } \\
\text { editor functions, }\end{array}$ & $\begin{array}{l}\text { parameter range : } \mathrm{x} \\
\min , \mathrm{x}_{\max }, \text { step, } \\
\text { input parameter } \\
\text { values: as value, as set } \\
\text { point vector }\left[\mathrm{x}_{\mathrm{i}}, \mathrm{t}_{\mathrm{i}}\right] \text {, } \\
\text { graphical input by } \\
\text { using a curve editor, }\end{array}$ \\
\hline $\begin{array}{l}\text { connecting } \\
\text { conditions } \\
\text { (predecessor / } \\
\text { successor) }\end{array}$ & $\begin{array}{l}\text { - placing of a } \\
\text { SegProgBox in a } \\
\text { experiment sequence } \\
\text { schema can be related to } \\
\text { predefined rules or } \\
\text { global time marks }\end{array}$ & time mark $t$ \\
\hline
\end{tabular}

Tab. 1: SegProgBox parameter type overview

A major set of SegProgBox parameters is directly connected via transformations to the high level parameters of the high level segments of the segment traces. SegProgBox parameters can be connected to more than one HLP of segments from different segment traces of the SegProgBox. Fig. 4 shows the principle of transformation of parameters of SegProgBoxes into the low level segments.

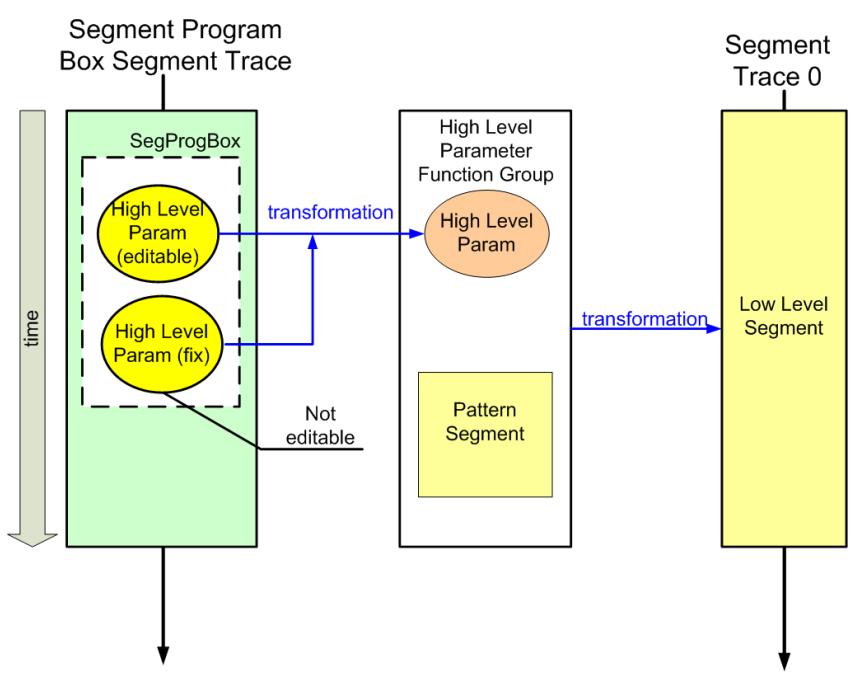

Fig. 4: Principle of two-stage transformation of the SegProgBox parameters into the low level segments

\section{SegProgBox Editor}


SegProgBoxes will be designed, created and tested by experts on demand. All SegProgBoxes are stored in topic oriented libraries. Only approved SegProgBoxes can be used by the experiment programmers to plan experiment runs or parts of it.

Design and modification of SegProgBoxes will be realized with a special editor tool (so called SegProgBox editor). Important preconditions are the existence of complete configurations of the involved components and the definition of HLP segment descriptions incl. their transformation functions, and constraints.

Because the knowledge about physical and technical dependencies will grown during the life span of the experiment the set of rules and dependencies will develop. In a first step it will always be possible to modify already successfully run SegProgBoxes.

\section{EXPERIMENT PROGRAM EDITING}

Important requirements for the editing process of experiment programs:

- The workflow and the tools should be applicable for both use cases: preparation of experiment programs and short term editing on existing experiment programs during the running experiment session.

- A reduction of complexity of the defined experiment description is very important.

- Expert knowledge of the segment control system must not be necessary for editing.

- A validation of the planned experiment program regarding feasibility is necessary.

- A preview of the planned experiment run based on the parameters of the SegProgBoxes should be possible.

- Reuse well-defined and successfully tested SegProgBoxes to simplify and speed-up the edit process. Furthermore this enhances the prospect of experiment success and the safety of experiment operation.

- Provide a physics oriented view of experiment programs to cover technical details.

- The experiment programming editor should be able to react to new requirements from the physics side for the design of experiments and should be able to handle configuration changes of W7-X components.

The experiment planning can be described in a four layer abstraction model shown in fig. 5 .

These levels are related to different views of the experiment planning process. Every level has its own special description elements for the behavior of components and their elements during experiments.
Abstrcation levels of experiment program definition process

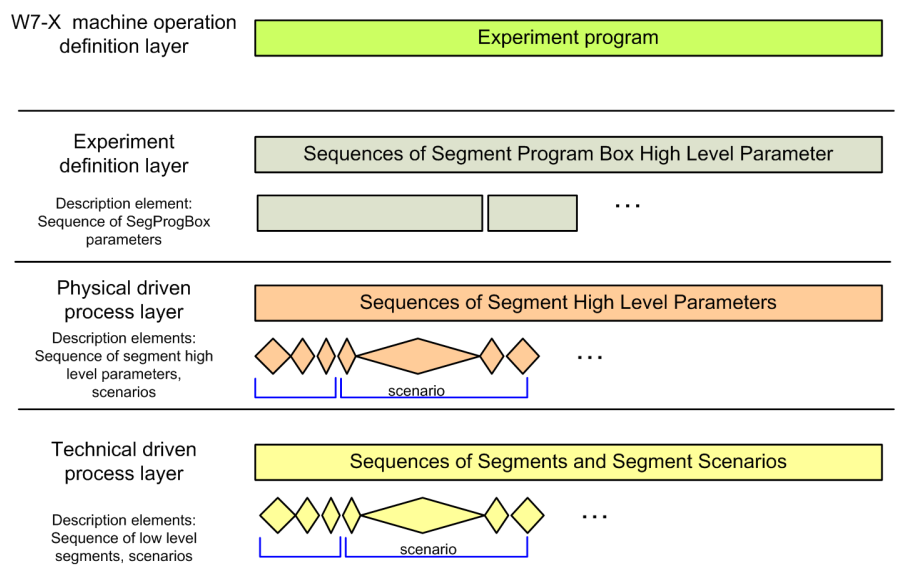

Fig. 5: Abstraction levels of the experiment planning process

The usual experiment planning is related to the experiment definition layer. This means that all experiment definitions are only based on data structures from type SegProgBox and their arrangement in the experiment program schedule.

The first step of the experiment planning work flow is to provide the proposals for the experimental phases. For W7-X plasma experiments five different phases have been defined: Experiment preparation phase, plasma build up phase, one or more Plasma experiment phases, plasma shut down phase, and post processing phase. For each phase a proposal has to be created by the requester (e.g. physicists, diagnostician). Especially for W7-X long pulse plasma operations a proposal for each plasma experiment and if necessary for each transition phase (describes the transition of machine and plasma between two experiment phases) is obligatory. These proposals are the interface between the experiment requesters and experiment planners. The necessary information of a proposal are collected in a formalized way. Extensive model calculation and simulations can be used for verification or for setting of machine or plasma parameters.

After the necessary proposals are completed and approved the experiment program editing can start. The main steps of this editing process are:

1. The actual configuration of the project determines the number and the types of all segment controlled components.

2. For the whole experiment run a time schedule has to be defined. A time schedule defines for example the number of phases and the corresponding time parameters (e.g. default duration, maximal duration). Every phase is connected to its appropriate proposal. Global time marks can be set to characterize special time points of the experiment run.

3. Insert SegProgBoxes into the experiment time schedule. The selection of a SegProgBox depends on the task at current experiment phase, which is described by a proposal. SegProgBoxes with defined connections to 
external time marks of time schedule will be placed in the right position automatically.

4. Modify time parameters (by time scaling the SegProgBox) and High Level parameters of the SegProgBox with respect to the specification of the proposals.

5. Global parameters of an experiment program defined into the proposal will be connected to the appropriate HLPs of the SegProgBox automatically.

6. If all areas of the experiment time schedule are completely described by SegProgBoxes the transformation of the SegProgBox parameters into high level segments of SegProgBoxes have to be processed.

7. Check completeness and consistency of the experiment program based on HLP segments of the components.

8. Preview the experiment run by using the HLP segments of the components in a simulator.

9. Transform the High Level segments into Low Level segments.

10. Assemble the group segments to appropriate project segments. Each segment switch in a segment trace requires the generation of a new project segment.

11. Compose project segments to scenarios that reflect the underlying proposals. There is one scenario for each proposal.

Fig.6 gives on overview about the structure of the W7-X experiment program at the different levels of experiment planning workflow.

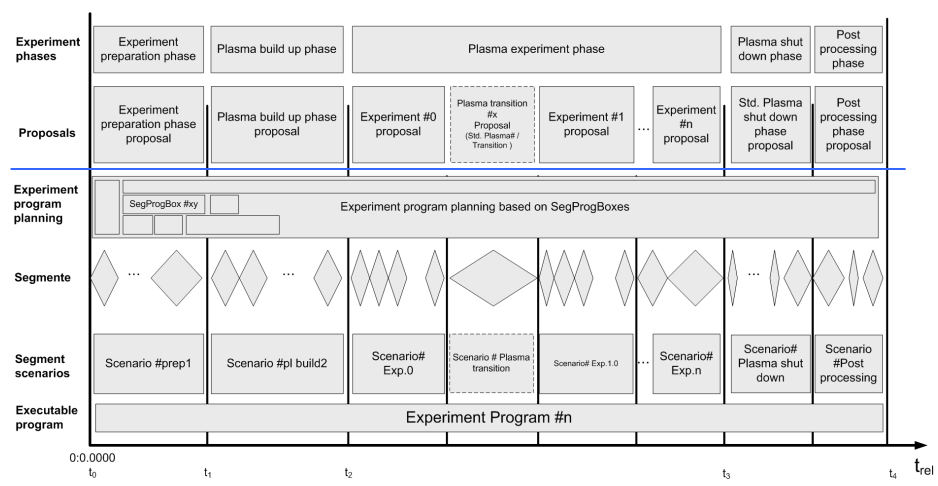

Fig. 6: Structure of an experiment program

\section{STATUS AND OUTLOOK}

There are two main aims in the further development of the experiment planning concept. The concept has to be enhanced and detail aspects of the workflow have to be discussed. Further more it is indispensable to find the adequate sets of High Level Parameters to describe the behavior of the segment controlled components at W7-X.

The second aim is to develop a workbench application. This workbench should be able to support all necessary steps of the workflow for editing experiment programs. HLPs for single segments already successfully implemented. A first prototype is on development as extension of the session leader program "Xcontrol" [7] allowing to display and change segment HLP of existing experiment programs. It will provide a graphical preview.

\section{ACKNOWLEDGEMENTS}

The authors would like to thank the XDV group and the W7-X $\mathrm{CoDaC}$ group for their support and for all helpful discussions.

\section{REFERENCES}

[1] J. Schacht, H. Laqua, M. Lewerentz, I. Müller, S. Pingel, A. Spring and A. Wölk, Overview and status of the control system of WENDELSTEIN 7-X, Fusion Engineering and Design, Volume 82, Issues 5-14, October 2007, Pages 988-994,

[2] H. Laqua, H. Niedermeyer, J. Schacht, A. Spring, Real Time Software for the Fusion Experiment WENDELSTEIN 7-X, Fusion Engineering and Design, Volume 82, Issues 5-14, October 2007,

[3] H. Riemann et al., From a physics discharge program to device controllinking the scientific and technical world at Wendelstein 7-X, SOFT conference, Rostock, Sept. 2009, will be published in Fusion engineering and design,

[4] J. Schacht, et al., Stellarator WEGA as a test-bed for the WENDELSTEIN 7-X control system concepts, Fusion Eng Des (2008), Volume 83, issues2-3, April 2008,

[5] J. Schacht, et al., Overview and status of the prototype project for Wendelstein 7-X control system, SOFT conference, Rostock, Sept. 2008, will be published in Fusion engineering and design,

[6] M. Lewerentz et al., First experiences with the new W7-X like control system at the WEGA Stellarator, SOFT conference, Rostock, Sept.2008, will be published in Fusion engineering and design,

[7] A. Spring, H. Laqua and H. Niedermeyer, User interaction concept for plasma discharge control on WENDELSTEIN 7-X, Fusion Engineering and Design, Volume 81, Issues 15-17, July 2006, Pages 1957-1961, 